\title{
Characterization of Near-Infrared and Raman Spectroscopy for In- Line Monitoring of a Low-Drug Load Formulation in a Continuous Manufacturing Process
}

Zachary D. Harms, * Zhenqi Shi, Rajesh A. Kulkarni, and David P. Myers

Small Molecule Design and Development, Eli Lilly and Company, Indianapolis, Indiana, 46285, United States

*E-mail: harms_zachary_d@lilly.com

Table of Contents

1. $\quad$ NIR spectra of $8 \%$ drug-load sample and pure API

S-3

2. Raman spectra of $8 \%$ drug-load sample and pure API

S-4

3. Table of PCA models for NIR data.

S-5

4. Table of PCA models for Raman data.

S-6

5. NIR PCA scores plots

S-7

6. Magnified view of NIR scores plot

S-8

7. Table of RSD for PCA scores distributions

S-9

8. Peak amplitude as a function of working distance

S-10 
Spectra and PCA model building. Representative NIR and Raman spectra are shown in Figures S1 and S2 respectively for both API and the pharmaceutical blend sample containing $8 \%(\mathrm{w} / \mathrm{w})$ of API and various excipients. These spectra are used to guide wavelength selection in the PCA model building. The wavelength regions in which there is a strong API absorbance and minimal interference from excipients are desirable. Several PCA models are built with a variety of pre-processing treatments and wavelength regions and are summarized in Table S1 for the NIR models and Table S2 for the Raman models. For both, the root mean square error of calibration (RMSEC) and the root mean square error of cross-validation (RMSECV) are used as metrics to evaluate model performance. All data are mean-centered and each model has 2 principle components (PC). Order of operations in spectral pre-processing follows order that each is listed in Tables S1 and S2, e.g. for 'SNV, $1^{\text {st }}$ Derivative' indicates that the spectra are first normalized by SNV followed by filtering by $2^{\text {nd }}$ derivative.

NIR Model Building. In Figure S1, the NIR spectrum for pure API (red dashed line) is overlaid with a blend sample containing 8\% (w/w) API (solid black line). There are two wavelength regions in which there is strong absorbance from the API, 1100-1300 nm and 1600-1750 nm. PCA models are built from each region separately, as well as models that combine the two wavelength regions. These results are summarized in Table S1. Furthermore, a variety of spectra pre-processing are also evaluated including standard normal variate (SNV) normalization, $1^{\text {st }}$ derivative filtering, $2^{\text {nd }}$ derivative filtering, extended mean scatter correction (EMSC), and Whittaker filtering. For each wavelength combination, $1^{\text {st }}$ derivative or $2^{\text {nd }}$ derivative pre-processing resulted in the lowest RMSEC and RMSECV, with $2^{\text {nd }}$ derivative having slightly lower RMSEC/RMSECV.

For wavelength selection in the NIR model, RMSEC decreases as wavelength range is targeted to spectral regions in which there is strong absorbance by the API. For $2^{\text {nd }}$ derivative pre-processing RMSEC was highest in which the entire wavelength region was used $(1100-2100 \mathrm{~nm})$ at 1.28E-5. As wavelength range decreases, RMSEC is 7.90E6 for $1104-1296 \mathrm{~nm}$ and $1600-1750 \mathrm{~nm}, 7.97 \mathrm{E}-6$ for $1600-1750 \mathrm{~nm}$, and $4.69 \mathrm{E}-6$ for $1104-1296 \mathrm{~nm}$. The region of 1104-1296 nm combined with $2^{\text {nd }}$ derivative pre-processing (Model 3) had the lowest RMSEC and was the final model used in the manuscript. Example scores plots for all four of these models are shown in Figure S3. In all models, there is considerable day-to-day variability. An example of day-to-day variability is shown in Figure S4. These data are a magnified view of Figure $2 b$ and show the difference in scores between two days (day 3 and 4 ) for the $8 \%$ drug load sample. The relative standard deviation of the scores distribution for each drug load on each day is used as an indication of within-day variability and these data are summarized in Table S3 for both NIR and Raman datasets.

Raman Model Building. In Figure S2, the Raman spectrum for pure API (red dashed line) is overlaid with a blend sample containing $8 \%(\mathrm{w} / \mathrm{w})$ API (solid black line). Here the blend sample is characterized by a fluorescent halo with a maximum at $500 \mathrm{~cm}^{-1}$. Background fluorescence is primarily from the microcrystalline cellulose (MCC) and intensity decreases as wavenumber increases. Several models are built that encompass a wide range of pre-processing and wavelength selection and these data are summarized in Table S2. For each wavelength range tested, $\mathrm{SNV}+2^{\text {nd }}$ derivative resulted in the lowest RMSEC and so was chosen as the pre-processing in the final model.

Three wavelength regions are analyzed; the entire wavelength region of $1890-150 \mathrm{~cm}^{-1}, 1700-1200 \mathrm{~cm}^{-1}$, and $1700-1575 \mathrm{~cm}^{-1}$. As wavelength region decreases, RMSEC increases. Scores plots for the three wavelength regions are shown in Figure 3. All models have the same pre-processing (SNV $+2^{\text {nd }}$ derivative). As wavelength region decreases, the separation between scores from different days also decreases. Minimizing day-to-day variability is desirable and the model with the smallest wavelength range, $1700-1575 \mathrm{~cm}^{-1}$, was chosen as the final model even though it has a higher RMSEC than the models with a wider wavelength range.

Raman Working Distance Optimization. In addition to optimization of PCA modeling, the arrangement of the Raman probe is also optimized and these data are summarized in Figure S5. Three non-contact optics are evaluated for the Raman probe and have spot sizes of 3, 4.7, and $6 \mathrm{~mm}$. The $3 \mathrm{~mm}$ optic had the most signal as compared to the 4.7 and $6 \mathrm{~mm}$ spot size optics. Within each optic, working distance was also optimized to increase signal. Working distance is defined as the distance between the powder level and the optic. The $3 \mathrm{~mm}$ spot size optic combined with a working distance of $10.2 \mathrm{~cm}$ provided the greatest signal and was selected for monitoring the pharmaceutical batch manufacturing process. 


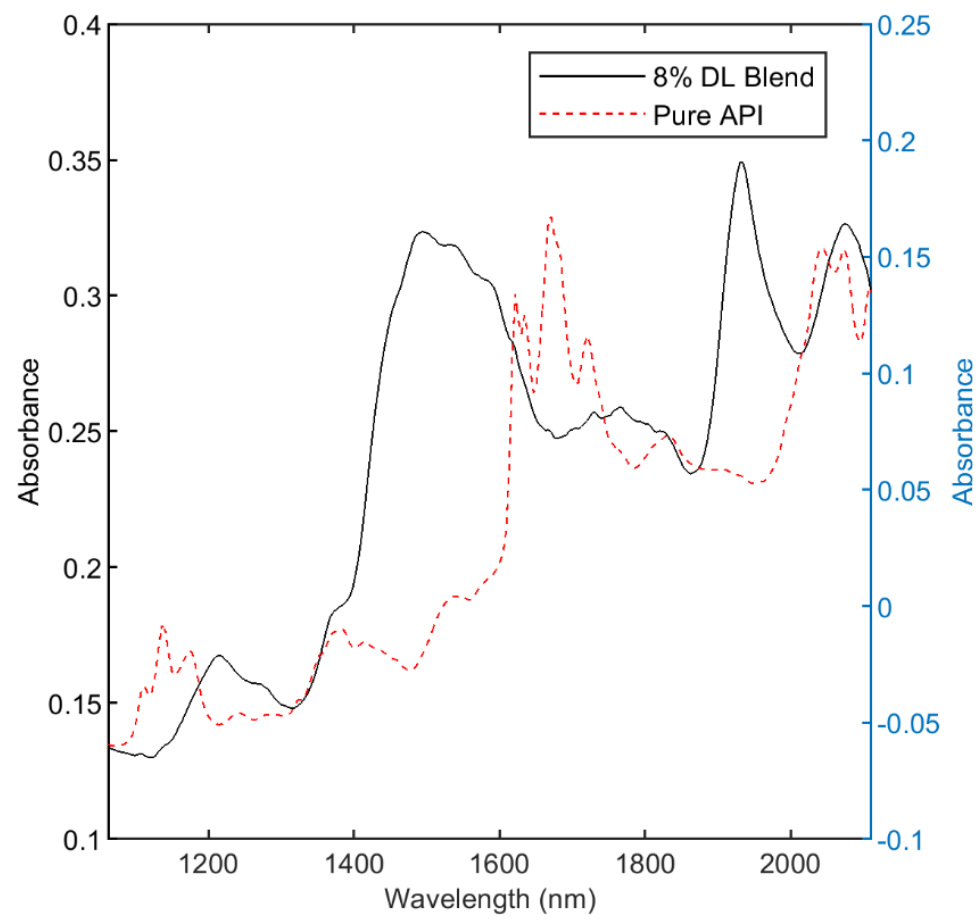

Figure S1. NIR spectrum of 8\% drug load pharmaceutical blend sample (left axis) and NIR spectrum of pure API sample (right axis). 


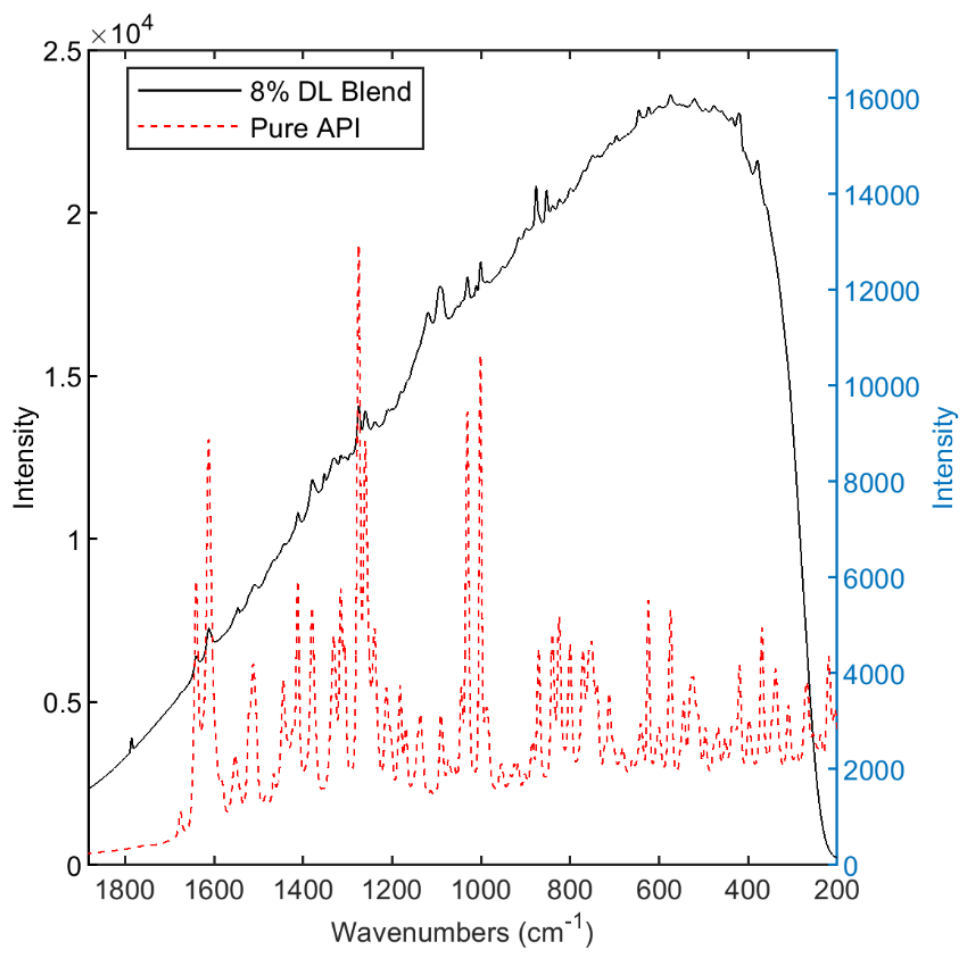

Figure S2. Raman spectrum of 8\% drug load pharmaceutical blend sample (left axis) and Raman spectrum of pure API sample (right axis). 


\begin{tabular}{|c|c|c|c|c|}
\hline Model Number & Pre-Processing & Wavelength Range (nm) & RMSEC & RMSECV \\
\hline 1 & SNV & $1104-1296$ & 7.99E-03 & $8.82 \mathrm{E}-03$ \\
\hline 2 & 1st derivative & $1104-1296$ & $3.77 \mathrm{E}-05$ & $4.98 \mathrm{E}-05$ \\
\hline 3 & 2nd derivative & $1104-1296$ & 4.69E-06 & $5.63 \mathrm{E}-06$ \\
\hline 4 & SNV, 1st derivative & $1104-1296$ & $1.29 \mathrm{E}-03$ & $1.57 \mathrm{E}-03$ \\
\hline 5 & SNV, 2nd derivative & $1104-1296$ & $4.50 \mathrm{E}-04$ & $5.29 \mathrm{E}-04$ \\
\hline 6 & SNV, Whittaker & $1104-1296$ & $9.25 \mathrm{E}-03$ & $1.11 \mathrm{E}-02$ \\
\hline 7 & SNV & $1100-2100$ & $1.79 \mathrm{E}-02$ & $2.32 \mathrm{E}-02$ \\
\hline 8 & 1st derivative & $1100-2100$ & $9.72 \mathrm{E}-05$ & $1.15 \mathrm{E}-04$ \\
\hline 9 & 2nd derivative & $1100-2100$ & $1.76 \mathrm{E}-05$ & $2.02 \mathrm{E}-05$ \\
\hline 10 & SNV, 1st derivative & $1100-2100$ & $1.42 \mathrm{E}-03$ & $1.65 \mathrm{E}-03$ \\
\hline 11 & SNV, 2nd derivative & $1100-2100$ & 3.04E-04 & 3.37E-04 \\
\hline 12 & SNV & $1100-2100$ & $1.29 \mathrm{E}-02$ & $1.58 \mathrm{E}-02$ \\
\hline 13 & SNV, EMSC & $1100-2100$ & $1.12 \mathrm{E}-02$ & $1.23 \mathrm{E}-02$ \\
\hline 14 & 2nd derivative & $1104-1710$ & $1.28 \mathrm{E}-05$ & $1.41 \mathrm{E}-05$ \\
\hline 15 & SNV, 2nd derivative & $1104-1710$ & $2.48 \mathrm{E}-04$ & $2.48 \mathrm{E}-04$ \\
\hline 16 & SNV & $1104-1710$ & $9.23 \mathrm{E}-03$ & $1.06 \mathrm{E}-02$ \\
\hline 17 & 1st derivative & $1104-1710$ & $6.22 \mathrm{E}-05$ & $6.90 \mathrm{E}-05$ \\
\hline 18 & SNV & $1104-1296$ and $1600-1750$ & $8.86 \mathrm{E}-03$ & $9.86 \mathrm{E}-03$ \\
\hline 19 & 1st derivative & $1104-1296$ and $1600-1750$ & $5.51 \mathrm{E}-05$ & 7.31E-05 \\
\hline 20 & 2nd derivative & $1104-1296$ and $1600-1750$ & 7.90E-06 & $9.25 \mathrm{E}-05$ \\
\hline 21 & SNV, 2nd derivative & $1104-1296$ and $1600-1750$ & $1.98 \mathrm{E}-04$ & $2.21 \mathrm{E}-04$ \\
\hline 22 & SNV, 2nd derivative & $1600-1750$ & $9.61 \mathrm{E}-04$ & $1.30 \mathrm{E}-03$ \\
\hline 23 & SNV & $1600-1750$ & $1.36 \mathrm{E}-02$ & $1.53 \mathrm{E}-02$ \\
\hline 24 & 1st derivative & $1600-1750$ & $2.62 \mathrm{E}-05$ & $3.75 \mathrm{E}-05$ \\
\hline 25 & 2nd derivative & $1600-1750$ & 7.97E-06 & $9.28 \mathrm{E}-06$ \\
\hline
\end{tabular}

Table S1. Summary of spectral pre-processing and wavelength selection for NIR PCA models. Final model used in manuscript is Model Number 3. All data are mean-centered and include two PCs. 


\begin{tabular}{|r|l|r|r|r|}
\hline Model Number & Pre-Processing & Wavelength Range $\left(\mathbf{c m}^{-1}\right)$ & RMSEC & RMSECV \\
\hline \hline 1 & SNV & $1700-1575$ & $2.12 \mathrm{E}-02$ & $2.30 \mathrm{E}-02$ \\
\hline 2 & 1st derivative & $1700-1575$ & $1.61 \mathrm{E}+00$ & $1.76 \mathrm{E}+00$ \\
\hline 3 & 2nd derivative & $1700-1575$ & $6.85 \mathrm{E}-01$ & $7.58 \mathrm{E}-01$ \\
\hline 4 & SNV, 1st derivative & $1700-1575$ & $2.99 \mathrm{E}-03$ & $4.88 \mathrm{E}-03$ \\
\hline 5 & SNV, 2nd derivative & $1700-1575$ & $1.19 \mathrm{E}-03$ & $1.46 \mathrm{E}-03$ \\
\hline 6 & SNV, Whittaker & $1700-1575$ & $2.22 \mathrm{E}-02$ & $2.44 \mathrm{E}-02$ \\
\hline 7 & SNV & $1890-150$ & $8.55 \mathrm{E}-03$ & $1.18 \mathrm{E}-02$ \\
\hline 8 & 1st derivative & $1890-150$ & $5.79 \mathrm{E}+00$ & $1.04 \mathrm{E}+01$ \\
\hline 9 & 2nd derivative & $1890-150$ & $1.45 \mathrm{E}+00$ & $1.67 \mathrm{E}+00$ \\
\hline 10 & SNV, 1st derivative & $1890-150$ & $7.53 \mathrm{E}-04$ & $1.36 \mathrm{E}-03$ \\
\hline 11 & SNV, 2nd derivative & $1890-150$ & $2.07 \mathrm{E}-04$ & $2.39 \mathrm{E}-04$ \\
\hline 12 & SNV, Whittaker & $1890-150$ & $5.08 \mathrm{E}-03$ & $6.02 \mathrm{E}-03$ \\
\hline 13 & SNV, EMSC & $1890-150$ & $7.66 \mathrm{E}-03$ & $2.05 \mathrm{E}-02$ \\
\hline 14 & SNV, 2nd derivative & $1700-1200$ & $3.93 \mathrm{E}-04$ & $4.11 \mathrm{E}-04$ \\
\hline
\end{tabular}

Table S2. Summary of spectral pre-processing and wavelength selection for Raman PCA models. Final model used in manuscript is Model Number 5. All data are mean-centered and include two PCs. 

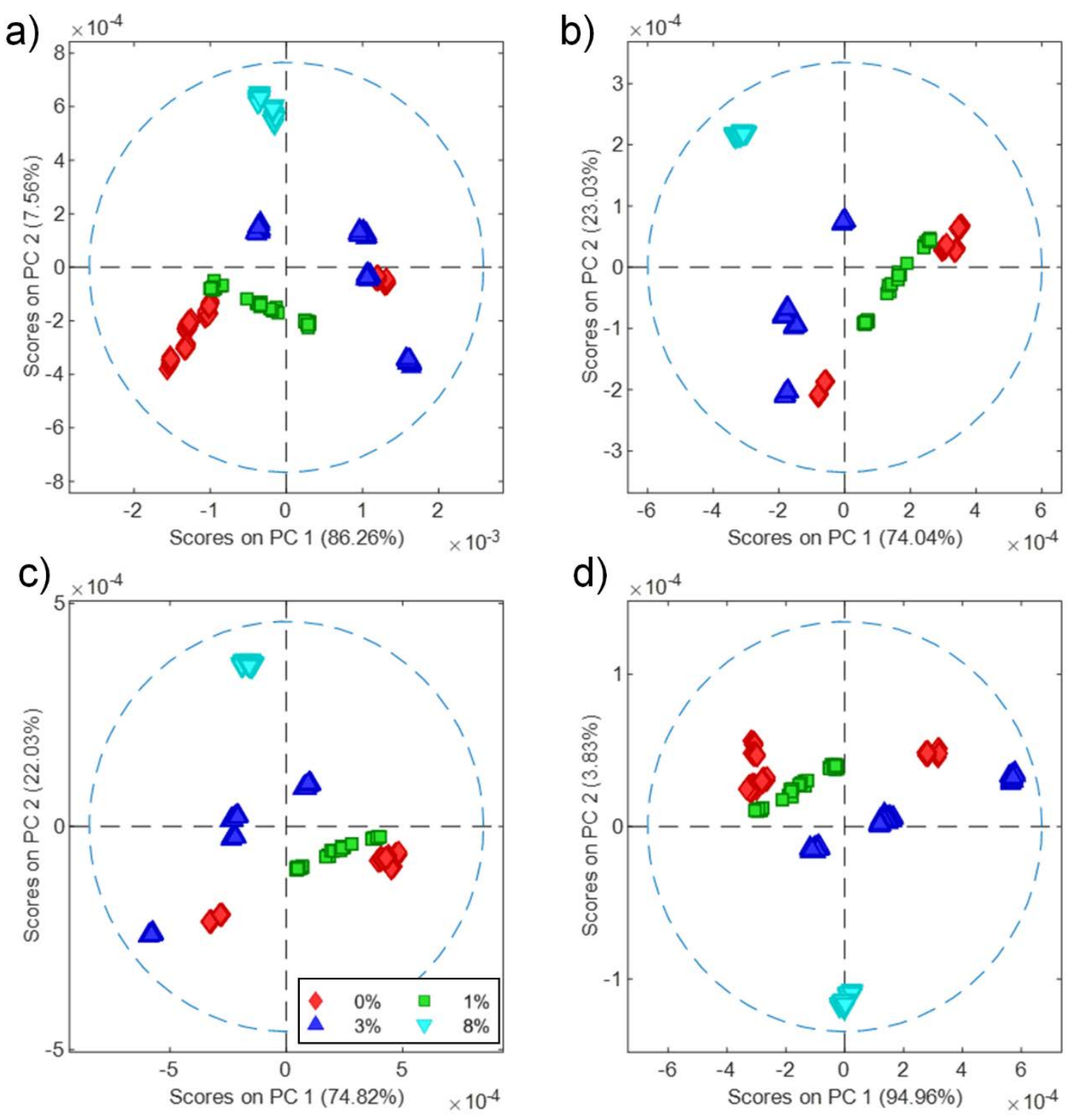

Figure S3. NIR PCA scores plots for four models. All four models are pre-processed by $2^{\text {nd }}$ derivative, but differ in the wavelength region used to build the model. In a) all wavelengths are used (Model Number 9 in Table S1), b) 1600-1750 nm (Model Number 25), and c) 11041296 and 1600-1750 nm (Model Number 20), and d) 1104-1296 nm (Model Number 3, final model used in analysis). Data are color-coded by API concentration in weight percent. 


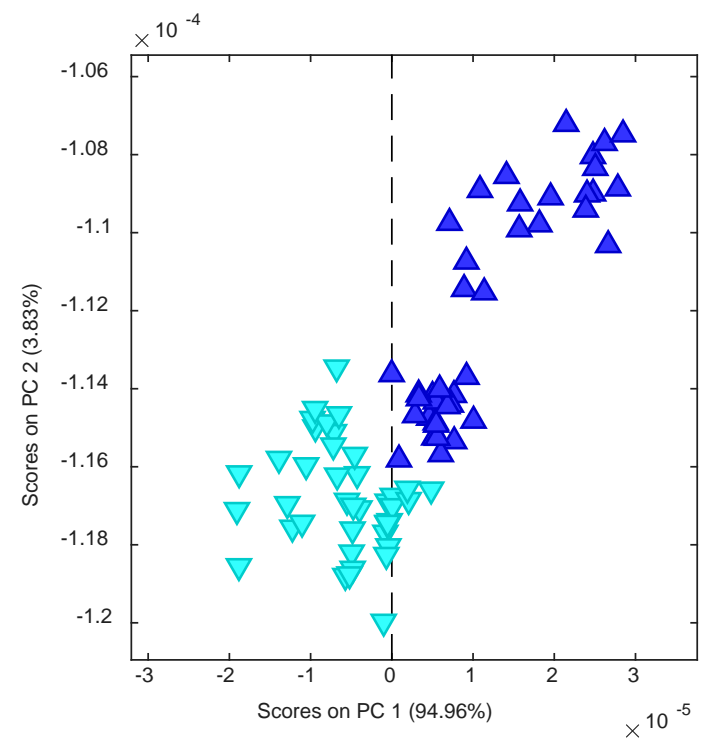

Figure S4. Magnified view of NIR scores plot in Figure 2b. View of Day 3 (dark blue triangles) and Day 4 (inverted light blue triangles) for $8 \%$ drug load samples. 


\begin{tabular}{|l|l|l|l|l|}
\hline & \multicolumn{2}{l|}{ NIR } & \multicolumn{2}{l|}{ Raman } \\
\hline \% API & Day 3 & Day 4 & Day 1 & Day 2 \\
\hline 0 & 67 & 15 & 55 & 77 \\
\hline 3 & 39 & 43 & 59 & 60 \\
\hline 8 & 48 & 69 & 64 & 54 \\
\hline
\end{tabular}

Table S3. Relative standard deviation (RSD) for average PCA scores distribution widths, $\bar{w}$, for NIR and Raman data. RSD is used as an indication of within-day variability. 


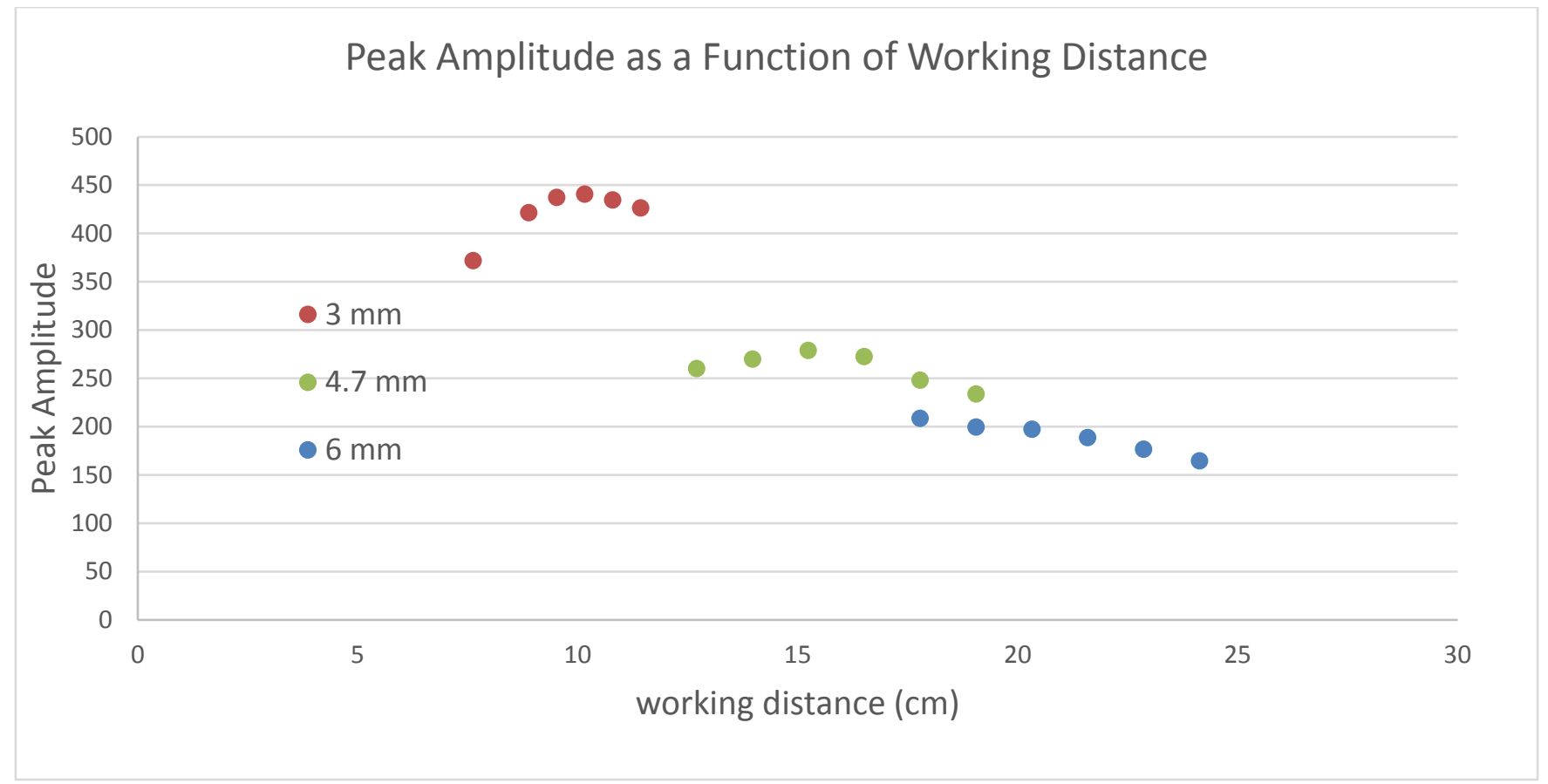

Figure S5. Peak amplitude at $1610 \mathrm{~cm}^{-1}$ as a function of working distance for the 3, 4.7, and $6 \mathrm{~mm}$ optics. Peak amplitude is at a maximum when working distance is $10.2,15.2$, and $20.3 \mathrm{~cm}$ for the $3,4.7$, and $6 \mathrm{~mm}$ optics, respectively 